\title{
The cross-sectional survey on COVID-19 vaccine hesitancy and it predictors among Chinese parents of 3-17 years aged children in Shenzhen City
}

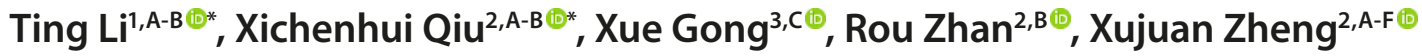 \\ ${ }^{1}$ Department of Obstetrics and Gynecology, South China Hospital, Health Science Center, Shenzhen University, Shenzhen, \\ Guangdong, China \\ ${ }^{2}$ School of Nursing, Health Science Centre, Shenzhen University, Shenzhen, Guangdong, China \\ ${ }^{3}$ School of Nursing, Southern Medical University, Guangzhou, Guangdong, China \\ A - Research concept and design, B - Collection and/or assembly of data, C - Data analysis and interpretation, \\ $D$ - Writing the article, E - Critical revision of the article, F - Final approval of the article
}

\footnotetext{
* Ting Li and Xichenhui Qiu contributed equally for this manuscript

Ting Li, Xichenhui Qiu, Xue Gong, Rou Zhan, Xujuan Zheng. The cross-sectional survey on COVID-19 vaccine hesitancy and it predictors among Chinese parents of 3-17 years aged children in Shenzhen City. Ann Agric Environ Med. 2022; 29(1): 120-125. doi: 10.26444/aaem/146263
}

\section{Abstract}

Introduction and Objective. Vaccinations programs on 3-17 years aged children in China have been launched in some cities since July 2021; and comparative evaluations are important to push the programs forward. Therefore, this study is conducted to explore the COVID-19 vaccine hesitancy and it predictors among Chinese parents of 3-17 years aged children; and their willingness to vaccinate their child/children.

Materials and method. A cross-sectional study was conducted based on the online survey; and 3484 participants were recruited in health centers of Shenzhen, China.

Results. The prevalence of COVID-19 vaccine hesitancy was $20.7 \%$ among Chinese parents of 3-17 years aged children; and 2976 participants (89.0\%) were reported to be willing or very willing to accept a COVID-19 vaccine for their child/children. Perceived trust degree of a COVID-19 vaccine (OR: 0.766), and perceived effectiveness of a COVID-19 vaccine (OR: 0.455) were negatively associated with vaccine hesitancy. Perceived risk degree of a COVID-19 vaccine (OR: 1.485) positively predicted vaccine hesitancy. Parents with a higher educational level (OR: 0.518) and working in public institution (OR: 0.741) had a lower level of vaccine hesitancy; whereas, parents with liberal professionals had a higher level of vaccine hesitancy (OR: 1.378).

Conclusions. These findings indicated that Chinese parents of 3-17 years aged children in Shenzhen City had a higher level of COVID-19 vaccine acceptability, compared with adults in Western counties. The significant factors of perceived trust degree, effectiveness, and risk degree of a COVID-19 vaccine, parents' educational level and occupation could predict COVID-19 vaccine hesitancy. The research results can be useful in supporting the development of effective and targeted children COVID-19 vaccination campaigns in China and worldwide.

\section{Keywords}

COVID-19; vaccine; vaccine hesitancy; Chinese parents; children; public health

\section{INTRODUCTION}

Since the outbreak of the coronavirus disease 2019 (COVID-19) pandemic, the infectious disease has caused the worldwide detrimental consequences on social and economic development, with negatively profound impacts on the work and daily life of everyone [1,2]. As of 22 November 2021, there have been over 256 million cases of COVID-19, including 5.2 million deaths, reported to World Health Organization (WHO) [3]. Even though the implementation of Public Health and Social Measures (PHSM) to limit the spread of COVID-19 such as mask wearing, limits and restrictions on public and private gatherings [4], vaccination still can be a key protective measure against COVID-19 to alleviate the devastating effect of the pandemic, and to reduce the

Address for correspondence: Xujuan Zheng, Shenzhen University E-mail: zhengxujuan@szu.edu.cn

Received: 20.12.2021; accepted: 28.01.2022; first published:08.02.2022 considerable burden of public health systems [5-7]. Up to November 2021, the vaccine candidates worldwide were 326 , of which 132 were in clinical testing [8].

As the generally acknowledged solution to preventing the coronavirus spread [9], the global herd immunity based on vaccination is dependent on not only vaccine safety and efficacy [10], but also individuals' willingness to be vaccinated [11]. An international survey across 19 countries reported that approximately $28.5 \%$ of participants would not consider taking a COVID-19 vaccine [12]. This hesitancy and refusal may alleviate the pandemic and put more pressure on health systems [13]. Previous studies already predicted that vaccine hesitancy could be a significant challenge for COVID-19 vaccine rollout $[14,15,16]$. The definition of vaccine hesitancy is the delay in acceptance or refusal of vaccination despite availability of vaccine services [17]. In recent years, vaccine hesitancy among not only citizens but also health professionals has become an emerging issue, as 
it could exacerbate vaccine refusal $[18,19]$. As one of the top 10 global health threats listed by WHO [20, 21], vaccine hesitancy varies across time, place, and type of vaccine, and is affected by various factors [22]. Thus, it is necessary to assess vaccine hesitancy of the COVID-19 vaccine and the factors that influence it in each country, in order to tailor educational activities to increase acceptance of the vaccine.

Since July 2021, vaccinations programs on 3-17 years aged children in China have been launched in some cities and may be covered the whole country step by step [23]. British research on COVID-19 vaccine described that parents were more likely to vaccinate themselves than their child/children, and the main concerns about children vaccination were as follows: not enough evidence, safety concerns, and the belief that children are hardly affected [24]. Actually, children can also become infected and transmit, and develop clinical complications from the COVID-19, so that there is an urgent need to approve children's COVID-19 vaccine uptake [25]. It is noted that the success of vaccinating these children of 3-17 age groups mainly relies on the parents' self-acceptance of COVID-19 vaccine and their willingness to vaccinate their child/children. However, little is known about COVID-19 vaccine hesitancy among Chinese parents of 3-17 years aged children and their willingness to vaccinate their child/ children. Moreover, it has been not clear that what factors could predict these parents' vaccine hesitancy.

COVID-19 vaccine hesitancy is an ongoing issue and a better understanding of its determinants, within the special population of parents with 3-17 years aged children, can be useful in supporting the development of effective and targeted children COVID-19 vaccination campaigns in China and worldwide [26]. Therefore, this study is conducted to explore the COVID-19 vaccine hesitancy and it predictors among Chinese parents of 3-17 years aged children; and their willingness to vaccinate their child/children.

\section{MATERIAL AND METHOD}

\section{Research design and participants}

The cross-sectional study was conducted based on the Sojump online survey platform. The participants were recruited when they had a physical examination in health centers of two hospitals in Shenzhen city. Recruitment was taken place from October to November of 2021 via recruiting posters and leaflets. The inclusion criteria were as follows: (1) being parents of 3-17 years aged children; (2) living in Shenzhen City (3) willing to participant this study; (4) being able to response the online questionnaires. The exclusion criteria were: parents or their children were with severe mental or physical illness. All participants were informed of the research purpose and process, and were assured of anonymity through the use of special code numbers to identify themselves. Informed consent was obtained from every respondent.

Parents meeting the inclusion criteria were invited to fill in the online questionnaire, which was produced, distributed, and collected with the online survey tool Sojump (http://www.sojump.com). Sojump is a professional online evaluation, survey, and voting platform that can offer humanized services including questionnaire design, data collection, and result reporting [27]. In order to increase the completed questionnaire quality and collect as many valid questionnaires as possible, electronic red envelopes of varying value were randomly distributed to improve respondents' initiative. In total of 3342 valid questionnaires were collected in the research.

\section{Measurements}

A socio-demographic questionnaire was developed by the researchers to collect data of participants' age, gender, nationality, registered residence, marital status, educational level, occupation, family income per month, the age of becoming a parent for the first time, and the number of children.

The aVHS (adult Vaccine Hesitancy Scale) was used to measure vaccine hesitancy. The ten-item instrument is based on a five-point Likert type scale, and the score of 1 represents the lowest degree of vaccine hesitancy and 5 the highest. The aVHS has seven items which are positively worded, and three items which are negatively worded. The total score of the tool ranges from 10-50, with each item scored from 1-5. The aVHS had been validated by the samples in the USA and China, and exhibits good internal consistency (Crohnbach's alpha $=0.82$ ) and validity, making it a reliable and valid tool for measuring vaccination uptake [10]. A score of 10 to 24 was categorized as "not hesitant", while a score of 25 to 50 was considered "hesitant" [10].

We also asked the participants to provide responses for the following items: willingness to get your child vaccinated against COVID-19, willingness to get your parents vaccinated against COVID-19, and willingness to get yourself vaccinated against COVID-19. The range of possible responses for these items was coded 1-5. Perceived trust degree of a COVID-19 vaccine, perceived risk degree of a COVID-19 vaccine, and perceived effectiveness of a COVID-19 vaccine were likewise asked to response; and the range of possible responses for the three items was coded 1-3. Moreover, whether the parents got vaccination against COVID-19; and whether the parents gave children any other self-funded vaccination (e.g. flu vaccine) were assessed in the study.

\section{Data analysis}

Data analyses were carried out by IBM SPSS Statistics, version 21. Descriptive statistics were used to describe the socio-demographic characteristics and other responses of participants by means (SD) for continuous data, and frequencies (proportions) for categorical data. The chi-square $(\chi 2)$ was used to detect any significant difference between various groups on the percentage of vaccine hesitancy. A multiple logistic regression analysis was used to identify the potential variables that can predict vaccine hesitancy. Multicollinearity was assessed via computing the variance inflation factor (VIF). The Hosmer and Lemeshow test was conducted to evaluate the goodness of fit of the model. The odds ratios (ORs) and 95\% confidence intervals (CIs) were used, and statistical significance was set at alpha $<0.05$.

\section{RESULTS}

\section{Participant profile}

Overall, 3484 participants responded to the survey, but 142 participants with incomplete data provided were excluded from data analysis. Finally, in total of 3342 questionnaires were included in the analysis set. The mean age of participants 
Table 1. The profile of participants $(n=3342)$

\begin{tabular}{|c|c|c|c|c|c|}
\hline \multirow[b]{2}{*}{ Variables } & \multirow{2}{*}{$\begin{array}{c}\mathrm{N}=3342 \\
\mathrm{n}(\%)\end{array}$} & \multicolumn{4}{|c|}{ Vaccine Hesitant } \\
\hline & & $\begin{array}{l}\text { Numbers } \\
\text { (n) }\end{array}$ & $\begin{array}{l}\text { Percentage } \\
(\%)\end{array}$ & $\begin{array}{c}\chi^{2} \\
\text { Value }\end{array}$ & $p$-Value \\
\hline \multicolumn{4}{|l|}{ Gender } & \multirow{3}{*}{2.367} & \multirow{3}{*}{0.124} \\
\hline Male (Father) & $1204(36.0)$ & 232 & 19.3 & & \\
\hline Female (Mother) & $2138(64.0)$ & 460 & 21.5 & & \\
\hline \multicolumn{4}{|l|}{ Nationality } & \multirow{3}{*}{2.048} & \multirow{3}{*}{0.152} \\
\hline Han nationality & $3192(95.5)$ & 654 & 20.5 & & \\
\hline Ethnic minority & $150(4.5)$ & 38 & 25.3 & & \\
\hline \multicolumn{4}{|l|}{ Registered residence } & \multirow{3}{*}{1.904} & \multirow{3}{*}{0.168} \\
\hline City residents & $1162(34.8)$ & 256 & 22.0 & & \\
\hline Floating population & $2180(65.2)$ & 436 & 20.0 & & \\
\hline \multicolumn{4}{|l|}{ Education } & \multirow{4}{*}{60.728} & \multirow{4}{*}{$<0.001^{*}$} \\
\hline High school or lower & $1300(38.9)$ & 206 & 15.9 & & \\
\hline University or college & $1898(56.8)$ & 426 & 22.4 & & \\
\hline Master or above & $144(4.3)$ & 60 & 41.7 & & \\
\hline \multicolumn{4}{|l|}{ Occupation } & \multirow{6}{*}{21.169} & \multirow{6}{*}{$<0.001^{*}$} \\
\hline Public institution & $612(18.3)$ & 96 & 15.7 & & \\
\hline Health professionals & $350(10.5)$ & 98 & 28.0 & & \\
\hline Liberal professionals & $872(26.1)$ & 186 & 21.3 & & \\
\hline Unemployed & $176(5.3)$ & 34 & 19.3 & & \\
\hline Others & $1332(39.8)$ & 278 & 20.9 & & \\
\hline \multicolumn{4}{|l|}{ Marital status } & \multirow{4}{*}{2.405} & \multirow{4}{*}{0.301} \\
\hline Married & 3224 (96.5) & 668 & 20.7 & & \\
\hline Divorced & $84(2.5)$ & 14 & 16.7 & & \\
\hline Single & $34(1.0)$ & 10 & 29.4 & & \\
\hline
\end{tabular}

Family income per

month (RMB)

\begin{tabular}{|c|c|c|c|c|c|}
\hline$<10000$ (USD < 1566) & $1326(39.7)$ & 256 & 19.3 & \multirow{3}{*}{9.862} & \multirow{3}{*}{$0.007^{*}$} \\
\hline $\begin{array}{l}10000-20000 \\
\text { (USD 1566-3132) }\end{array}$ & $1166(34.9)$ & 228 & 19.6 & & \\
\hline$>20000$ (USD >3132) & $850(25.4)$ & 208 & 24.5 & & \\
\hline \multicolumn{4}{|l|}{ Number of children } & \multirow{5}{*}{6.545} & \multirow{5}{*}{0.088} \\
\hline 1 & $1152(34.5)$ & 234 & 20.3 & & \\
\hline 2 & $1886(56.4)$ & 378 & 20.0 & & \\
\hline 3 & $254(7.6)$ & 66 & 26.0 & & \\
\hline 4 or more & $50(1.5)$ & 14 & 28.0 & & \\
\hline
\end{tabular}

Age of becoming a

parent for the first time

\begin{tabular}{lccc}
\hline $18-22$ years & $270(8.1)$ & 52 & 19.3 \\
\hline $23-25$ years & $1026(30.7)$ & 166 & 16.2 \\
\hline $26-30$ years & $1514(45.3)$ & 336 & 22.2 \\
\hline$\geq 31$ years & $532(15.9)$ & 138 & 25.9 \\
\hline
\end{tabular}

Whether parents gave

children any other self-

funded vaccination

$3.302 \quad 0.069$

\begin{tabular}{|c|c|c|c|}
\hline Yes & $2354(70.4)$ & 468 & 19.9 \\
\hline No & $988(29.6)$ & 224 & 22.7 \\
\hline
\end{tabular}

Whether parents got

vaccination against

COVID-19

$44.939<0.001^{*}$

\begin{tabular}{cccc}
\hline Yes & $3216(96.2)$ & 636 & 19.8 \\
\hline No & $126(3.8)$ & 56 & 44.4 \\
\hline
\end{tabular}

was $35.35 \pm 4.95$ years, and more than half of them $(64.4 \%)$ were mothers. The participants' profile is summarized in Table 1. A significantly higher proportion of participants, who were health professionals, with a higher educational level, with a higher family income, aged older than 31 years, and not got vaccination against COVID-19, had a higher level of COVID-19 vaccine hesitant $(\boldsymbol{p}<0.05)$.

\section{Willingness and attitude of participants regarding COVID-19 vaccination}

In this study, 2976 participants (89.0\%) were reported to be willing or very willing to accept a COVID-19 vaccine for their child/children; while 66 participants (2.0\%) were reported to be reluctant or very reluctant to accept it for children. In terms of a COVID-19 vaccine for their elderly parents, 3162 participants (94.6\%) were reported to be willing or very willing; and 38 participants (1.1\%) were reported to be reluctant or very reluctant. Willingness and attitude of participants regarding COVID-19 vaccination were shown in Table 2.

Table 2. Willingness and attitude of participants regarding COVID-19 vaccination $(n=3342)$

Variables Frequency $(\mathrm{n}) \quad$ Percentage $(\%)$

Willingness to get your child vaccinated against

COVID-19

\begin{tabular}{lcc}
\hline Very willing & 1554 & 46.5 \\
\hline Willing & 1422 & 42.5 \\
\hline General & 300 & 9.0 \\
\hline Reluctant & 54 & 1.6 \\
\hline Very reluctant & 12 & 0.4 \\
\hline $\begin{array}{l}\text { Willingness to get yourself vaccinated against } \\
\text { COVID-19 }\end{array}$ & \\
\hline Very willing & 1760 & 52.7 \\
\hline Willing & 1448 & 43.3 \\
\hline General & 124 & 3.7 \\
\hline Reluctant & 4 & 0.1 \\
\hline Very reluctant & 6 & 0.2 \\
\hline
\end{tabular}

Willingness to get your parents vaccinated against COVID-19

\begin{tabular}{lcc}
\hline Very willing & 1672 & 50.1 \\
\hline Willing & 1490 & 44.6 \\
\hline General & 142 & 4.2 \\
\hline Reluctant & 30 & 0.9 \\
\hline Very reluctant & 8 & 0.2 \\
\hline Perceived trust degree of a COVID-19 vaccine & & \\
\hline Very trust/trust & 3086 & 92.3 \\
\hline General & 240 & 7.2 \\
\hline Very distrust/distrust & 16 & 0.5 \\
\hline Perceived risk degree of a COVID-19 vaccine & & \\
\hline Low & 2804 & 83.9 \\
\hline Medium & 442 & 13.2 \\
\hline High & 96 & 2.9 \\
\hline Perceived effectiveness of a COVID-19 vaccine & & \\
\hline Very effective & 1310 & 39.2 \\
\hline Effective & 1698 & 50.8 \\
\hline General & 334 & 10.0 \\
\hline
\end{tabular}




\section{Responses to questions about vaccine hesitancy among participants}

In total of 2650 participants $(79.3 \%)$ with a score of 10 to 24 as measured by the aVHS were categorized as vaccine acceptance, while 692 participants $(20.7 \%)$ with a score of 25-50 were considered as vaccine hesitancy. The mean scores of ten items in the aVHS were described in Table 3.

Table 3. The mean scores of ten items in the aVHS

\begin{tabular}{lc}
\hline Items & Mean \pm SD \\
\hline 1. Vaccines are important for my health & $1.69 \pm 0.95$ \\
\hline 2. Vaccines are effective & $1.75 \pm 0.93$ \\
\hline $\begin{array}{l}\text { 3. Being vaccinated is important for the health of others in my } \\
\text { community. }\end{array}$ & $1.68 \pm 0.93$ \\
\hline 4. All routine vaccinations recommended by the CDC are beneficial & $1.64 \pm 0.92$ \\
\hline 5. New vaccines carry more risks than older vaccines. & $3.09 \pm 1.24$ \\
\hline $\begin{array}{l}\text { 6. The information I receive about vaccines from the CDC is reliable } \\
\text { and trustworthy. }\end{array}$ & $1.76 \pm 0.82$ \\
\hline 7. Getting vaccines is a good way to protect me from disease. & $1.66 \pm 0.80$ \\
\hline $\begin{array}{l}\text { 8. Generally, I do what my doctor or healthcare provider } \\
\text { recommends about vaccines for me. }\end{array}$ & $1.63 \pm 0.79$ \\
\hline 9. I am concerned about serious adverse effects of vaccines. & $3.08 \pm 1.16$ \\
\hline 10. I do not need vaccines for diseases that are not common anymore. & $2.61 \pm 1.27$ \\
\hline
\end{tabular}

\section{Predictors of COVID-19 vaccine hesitancy}

The results of logistic regression analysis were showed in Table 4. Perceived trust degree of a COVID-19 vaccine (OR: 0.766), and perceived effectiveness of a COVID-19 vaccine (OR: $0.455)$ were negatively associated with COVID-19 vaccine hesitancy. Perceived risk degree of a COVID-19 vaccine (OR: 1.485) positively predicted COVID-19 vaccine hesitancy. Parents with a higher educational level (OR: 0.335, 0.518),

Table 4. Significant factors predicting COVID-19 vaccine hesitancy

\begin{tabular}{|c|c|c|c|c|c|c|}
\hline Variables & $\begin{array}{c}B \\
\text { Value }\end{array}$ & $\begin{array}{c}S E \\
\text { value }\end{array}$ & $\begin{array}{c}\text { Wald } c^{2} \\
\text { value }\end{array}$ & $p$-Value & OR & $95 \% \mathrm{Cl}$ \\
\hline $\begin{array}{l}\text { Perceived trust } \\
\text { degree of a } \\
\text { COVID-19 vaccine }\end{array}$ & -0.266 & 0.115 & 5.416 & 0.020 & 0.766 & $0.612-0.959$ \\
\hline $\begin{array}{l}\text { Perceived risk } \\
\text { degree of a } \\
\text { COVID-19 vaccine }\end{array}$ & 0.395 & 0.071 & 30.599 & $<0.001$ & 1.485 & $1.291-1.708$ \\
\hline $\begin{array}{l}\text { Perceived } \\
\text { effectiveness of a } \\
\text { COVID-19 vaccine }\end{array}$ & -0.787 & 0.101 & 60.433 & $<0.001$ & 0.455 & $0.373-0.555$ \\
\hline \multicolumn{7}{|l|}{ Education } \\
\hline $\begin{array}{l}\text { University } \\
\text { or college }\end{array}$ & -1.093 & 0.242 & 20.477 & $<0.001$ & 0.335 & $0.209-0.538$ \\
\hline Master or above & -0.659 & 0.213 & 9.523 & 0.002 & 0.518 & $0.341-0.786$ \\
\hline \multicolumn{7}{|l|}{ Occupation } \\
\hline Public institution & -0.300 & 0.147 & 4.175 & 0.041 & 0.741 & $0.556-0.988$ \\
\hline $\begin{array}{l}\text { Liberal } \\
\text { professionals }\end{array}$ & 0.321 & 0.122 & 6.920 & 0.009 & 1.378 & $1.085-1.750$ \\
\hline \multicolumn{7}{|l|}{$\begin{array}{l}\text { Whether got } \\
\text { vaccination against } \\
\text { COVID-19 }\end{array}$} \\
\hline Yes & 92 & 0.214 & 28 & 01 & 399 & \\
\hline
\end{tabular}

$\begin{array}{lllllllllll} & \text { Yes } & & 0.399 & 0.2620 .606\end{array}$ not getting COVID-19 vaccination working in public institution (OR: 0.741), and got vaccination against COVID-19 (OR: 0.399) had a lower level of vaccine hesitancy; whereas, parents with liberal professionals had a higher level of vaccine hesitancy (OR: 1.378).

\section{DISCUSSION}

A vaccine is well recognized to be one of the most powerful and cost-effective methods for diminishing community spread of COVID-19 in large population $[10,28]$. However, COVID-19 vaccine hesitancy among not only citizens but also health professionals has become an emerging issue [18, 19]. An international survey reported that COVID-19 vaccine acceptance has high heterogeneity across different countries; therefore, it is important to clarify the acceptance of a vaccine in each country or region [12]. Nowadays, COVID-19 vaccinations programs on 3-17 years aged children in China have been launched in some cities [23]. To the best of my knowledge, this is the first study to explore the predictors of COVID-19 vaccine hesitancy among Chinese parents of 3-17 years aged children, and their willingness to vaccinate their child/children. The research results can be useful in supporting the development of effective and targeted children COVID-19 vaccination campaigns in China and worldwide, by a better understanding of vaccine hesitancy and its predictors, within the special population of parents with 3-17 years aged children.

In this study, we found that the prevalence of COVID-19 vaccine acceptability in Shenzhen City was $79.3 \%$ among Chinese parents of 3-17 years aged children, much higher than the previous study findings from other countries. For instance, a recently study explored the current COVID-19 vaccine acceptability and hesitancy situation in Japan, and found that $62.1 \%$ of Japanese adults would be willing to receive a COVID-19 vaccine [28]. It has been reported that the prevalence of vaccine acceptability in Europe approximately ranged from $60.0 \%$ to $78.0 \%$ [29, 30, 31, 32], i.e., COVID-19 vaccine hesitancy was $31.1 \%$ in Italy [20] and 38.0\% in France [32]. Previous research in the U.S. showed that 25.0\%-43.1\% of respondents reported to be hesitant $[33,34,35]$. These findings indicated that Chinese parents of 3-17 years aged children in Shenzhen City had a higher level of COVID-19 vaccine acceptability, compared with adults in Western counties.

In this study, 2976 participants (89.0\%) were reported to be willing or very willing to accept a COVID-19 vaccine for their child/children; 3162 participants (94.6\%) were reported to be willing or very willing to accept for an elderly parent; and 3208 participants $(96.0 \%)$ were reported to be willing or very willing to accept a COVID-19 vaccine for themselves. The findings were well aligned with the previous studies $[13,24]$ that indicated parents were more cautious about COVID-19 vaccine for their child/children and less likely to vaccine their child/children than their parents or themselves. Therefore, more explanations need to be conducted to alleviate parents' concerns about COVID-19 vaccine for children.

Our research found that parents' attitudes of safety and effectiveness regarding COVID-19 vaccination played an important role in COVID-19 vaccination acceptability and hesitancy. For instance, perceived trust degree of a COVID-19 vaccine (OR: 0.766), and perceived effectiveness of a COVID-19 vaccine (OR: 0.455$)$ were positively associated 
with COVID-19 vaccine acceptability; while perceived risk degree of a COVID-19 vaccine (OR: 1.485) positively predicted COVID-19 vaccine hesitancy. Prior study likewise described that parents showed a substantial shift in attitudes towards the COVID- 19 vaccine based on safety and effectiveness [13]. To be specific, the majority of the respondents would accept a vaccine with high levels of safety and effectiveness, but only one-third of them would accept a vaccine with lower levels of safety and effectiveness [13]. Unlike previous vaccines with years or decades of clinical trials, COVID-19 vaccines were developed at "wrap speed" [36]. Therefore, parents' concerns over the speed of its development and concerns about safety and effectiveness may be even more prominent for the COVID-19 vaccine. According to the multiple regression analysis, parents with a higher educational level (OR: 0.518) had a lower level of vaccine hesitancy. By contrast, the univariate analysis in Table 1 presented that a significantly higher proportion of participants with a higher educational level had a higher level of COVID-19 vaccine hesitant $(\boldsymbol{p}<0.05)$. The influence of confounding factors in univariate analysis and the most parents having higher education in the study could lead to the opposite results. In the present research, occupation of parents was reported to predict COVID-19 vaccine hesitancy. For example, parents working in public institution (OR: 0.741) had a lower level of vaccine hesitancy; whereas, parents with liberal professionals had a higher level of vaccine hesitancy (OR: 1.378). These findings indicated that health professionals should pay more attention to these parents likely to have relatively high COVID-19 hesitancy levels in this context: Chinese parents of 3-17 years aged children with lower education level, and parents with liberal professionals.

Some limitations need to be noted. Firstly, the crosssectional surveys are to only provide researchers with a snapshot of a sample of population at a single point in time, and cannot enable researchers to capture any changes in COVID-19 vaccine acceptability and hesitancy over time. Secondly, as in studies of this nature, the respondents were the parents who were willing to respond to the questionnaire; and some less motivated parents or those who are less willing to get vaccine were prone to be non-respondents that could lead to a bias in the study. Thirdly, owing to the time and financial limitations, the study focused on parents of 3-17 years aged children merely from one city, which could cause that the group surveyed was not representative of all Chinese parents. In consideration of regional diversity, this study could be replicated in other cities or regions of China.

\section{CONCLUSION}

Vaccinations programs on 3-17 years aged children in China have been launched in some cities since July 2021; and comparative evaluations are important to push the programs forward. COVID-19 Vaccine hesitancy is an ongoing issue and a better understanding of its determinants, within the special population of parents with 3-17 years aged children, can be useful in supporting the development of effective and targeted children COVID-19 vaccination campaigns in China and worldwide. Therefore, this study is conducted to explore the COVID-19 vaccine hesitancy and it predictors among Chinese parents of 3-17 years aged children; and their willingness to vaccinate their child/children. Research found the prevalence of COVID-19 vaccine hesitancy was $20.7 \%$ among Chinese parents of 3-17 years aged children in Shenzhen City. Among these parents, 2976 participants $(89.0 \%)$ were reported to be willing or very willing to accept a COVID-19 vaccine for their child/children. These findings indicated that Chinese parents of 3-17 years aged children in Shenzhen City had a higher level of COVID-19 vaccine acceptability, compared with adults in Western counties. The significant factors of perceived trust degree, effectiveness, and risk degree of a COVID-19 vaccine, parents' educational level and occupation could predict COVID-19 vaccine hesitancy among Chinese parents of 3-17 years aged children.

\section{Acknowledgements|}

This study was funded by the General Program of Stable Support Plan for Universities in Shenzhen City (20200804101756002).

\section{REFERENCES}

1. Chriscaden K. Impact of COVID-19 on People's Livelihoods, Their Health and Our Food Systems. WHO, 2020. https://www. http://www. ilo.org/global/about-the-ilo/newsroom/statements-and-speeches/ WCMS_757974/1

ang--en/index.htm (access: 2021.11.13).

2. Dong E, Du H, Gardner L. An interactive web-based dashboard to track COVID-19 in real time. Lancet Infect Dis. 2020; 20: 533-534. https:// doi.org/10.1016/S1473-3099(20)30120-1.

3. WHO Coronavirus Disease (COVID-19) Dashboard. https://covid19. who.int (access: 2021.11.22).

4. Public Health and Social Measures (PHSM). WHO, 2021. https:// covid19.who.int (access: 2021.11.22).

5. Qualls N, Levitt A, Kanade N, et al. CDC Community Mitigation Guidelines Work Group. Community Mitigation Guidelines to Prevent Pandemic Influenza-United States, 2017. MMWR Recomm Rep. 2017; 66: 1-34. https://doi.org/ 10.15585/mmwr.rr6601al.

6. Gori D, Reno C, Remondini D, et al. Are We Ready for the Arrival of the New COVID-19 Vaccinations? Great Promises and Unknown Challenges Still to Come. Vaccines. 2021; 9: 173. https://doi.org/ $10.3390 /$ vaccines 9020173

7. Fernandes N, Costa D, Costa D, et al. Predicting COVID-19 Vaccination Intention: The Determinants of Vaccine Hesitancy. Vaccines. 2021; 9: 1161. https://doi.org/10.3390/vaccines9101161

8. COVID-19 vaccine tracker and landscape. WHO, 2021. https://www. who.int/publications/m/item/draft-landscape-of-covid-19-candidatevaccines (access: 2021.11.20).

9. Fan YJ, Chan KH, Hung IFN. Safety and Efficacy of COVID-19 Vaccines: A Systematic Review and Meta-Analysis of Different Vaccines at Phase 3. Vaccines. 2021; 9: 989. https://doi.org/10.3390/vaccines 9050467

10. Akel KB, Masters NB, Shih SF, et al. Modification of a Vaccine Hesitancy Scale for use in adult vaccinations in the United States and China. Hum Vaccin Immunother. 2021; 17(8):1-8. https://doi.org/10.1080/2 1645515.2021.1884476

11. Thunstrom L, Ashworth M, Finnoff D, et al. Hesitancy towards a COVID-19 vaccine and prospects for herd immunity. SSRN. 2020; 1-51. https://doi.org/ 10.2139/ssrn.3593098

12. Randall T, Sam C, Tartar A, et al. More Than 108 Million Shots Given: Covid-19 Tracker. https://www.bloomberg.com/graphics/covidvaccine-tracker-global-distribution/ (access: 2021.11.08).

13. Lu J, Wen X, Guo Q, et al. Sensitivity to COVID-19 Vaccine Effectiveness and Safety in Shanghai, China. Vaccines. 2021; 9: 472. https://doi. org/10.3390/vaccines 9050472

14. Barello S, Nania T, Dellafiore F, et al. "Vaccine hesitancy" among university students in Italy during the COVID- 19 pandemic. Eur J Epidemiol. 2020; 1: 3. https://doi.org/ 10.1007/s10654-020-00670-Z

15. Fu C, Wei Z, Pei S, et al. Acceptance and preference for COVID-19 vaccination in health-care workers (HCWs). medRxiv. 2020,20060103. https://doi.org/ 10.1101/2020.04.09.20060103

16. Khan YH, Mallhi TH, Alotaibi NH, et al. Threat of COVID-19 vaccine hesitancy in Pakistan: the need for measures to neutralizeleading narratives. Am J Trop Med Hyg. 2020; 103(2): 603-04. https://doi. org/10.4269/ajtmh.20-0654.18 
17. MacDonald NE, Eskola J, Liang X. Vaccine hesitancy: definition, scope and determinants. Vaccine. 2015; 33(34): 4161-64. https://doi. org/10.1016/j.vaccine.2015.04.036

18. Di Martino G, Di Giovanni P, Di Girolamo A, et al. Knowledge and Attitude towards Vaccination among Healthcare Workers: A Multicenter Cross-Sectional Study in a Southern Italian Region. Vaccine. 2020; 8: 248. https://doi.org/10.3390/vaccines8020248

19. Karafillakis E, Dinca I, Apfel F, et al. Vaccine hesitancy among healthcare workers in Europe: A qualitative study. Vaccine. 2016; 34 5013-5020. https://doi.org/10.1016/j.vaccine.2016.08.029

20. Reno C, Maietti E, Fantini MP, et al. Enhancing COVID-19 Vaccines Acceptance: Results from a Survey on Vaccine Hesitancy in Northern Italy. Vaccine. 2021; 9: 378. https://doi.org/10.3390/vaccines9040378

21. World Health Organization. Ten Threats to Global Health in 2019. https://www.who.int/news-room/spotlight/ten-threats-to-globalhealth-in-2019 (access: 2021.11.08).

22. MacDonald NE. Vaccine hesitancy: Definition, scope and determinants. Vaccine. 2015; 33: 4161-4164. https://doi.org/ 10.1016/j. vaccine.2015.04.036

23. The COVID-19 vaccine available for ages $3-17$ is coming. https://m. thepaper.cn/baijiahao_13643524 (access: 2021.11.20).

24. Bell S, Clarke R, Mounier-Jack S, et al. Parents' and guardians' views on the acceptability of a future COVID-19 vaccine: A multi-methods study in England. Vaccine. 2020; 38: 7789-7798. https://doi.org/10.1016/j. vaccine.2020.10.027

25. Anderson EJ, Campbell JD, Creech CB, et al. Warp speed for COVID-19 vaccines: Why are children stuck in neutral? Clin Infect Dis. 2020; 73: 336-340. https://doi.org/ 10.1093/cid/ciaa1425

26. Rapid Literature Review on Motivating Hesitant Population Groups in Europe to Vaccinate. https://www.ecdc.pa.eu/en/publications-data/ rapid-literature-review-motivating-hesitant-population-groupseurope-vaccinate (access: 2021.11.13).

27. Zhang XT, Wen D, Liang J, et al. How the public uses social media wechat to obtain health information in china: a survey study. BMC Med Inform Decis. 2017; 17(Suppl 2): 66. https://doi.org/10.1186/s12911017-0470-0
28. Machida M, Nakamura I, Kojima T, et al. Acceptance of a COVID-19 Vaccine in Japan during the COVID-19 Pandemic. Vaccine. 2021; 9: 210. https://doi.org/ 10.3390/vaccines 9030210

29. Barello S, Nania T, Dellafiore F, et al. 'Vaccine hesitancy' among university students in Italy during the COVID-19 pandemic. Eur J Epidemiol. 2020; 35: 781-783. https://doi.org/ 10.1007/s10654-02000670-Z

30. Gagneux-Brunon A, Detoc M, Bruel S, et al. Intention to get vaccinations against COVID-19 in French healthcare workers during the first pandemic wave: A cross-sectional survey. J Hosp Infect. 2021; 108: 168-173. https://doi.org/10.1016/j.jhin.2020.11.020

31. Murphy J, Vallieres F, Bentall RP, et al. Psychological characteristics associated with COVID-19 vaccine hesitancy and resistance in Ireland and the United Kingdom. Nat Commun. 2021; 12: 29. https://doi. org/10.1038/s41467-020-20226-9

32. Neumann-Bohme S, Varghese NE, Sabat I, et al. Once we have it, will we use it? A European survey on willingness to be vaccinated against COVID-19. Eur J Health Econ. 2020; 21: 977-982. https://oi.org/ 10.1007/s10198-020-01208-6

33. Malik AA, McFadden SM, Elharake J, et al. Determinants of COVID-19 Vaccine Acceptance in the US. E Clinical Medicine. 2020; 26: 100495. https://doi.org/10.1016/j.eclinm.2020.100495

34. Fisher KA, Bloomstone SJ, Walder J, et al. Attitudes Toward a Potential SARS-CoV-2 Vaccine: A Survey of U.S. Adults. Ann Intern Med. 2020; 173: 964-973. https://doi.org/ 10.7326/M20-3569

35. Reiter PL, Pennell ML, Katz ML. Acceptability of a COVID-19 Vaccine among Adults in the United States: How Many People Would Get Vaccinated? Vaccine. 2020; 38: 6500-6507. https://doi.org/ 10.1016/j. vaccine.2020.08.043

36. Zhu FC, Guan XH, Li YH, et al. Immunogenicity and safety of a recombinant adenovirus type-5-vectored COVID-19 vaccine in healthy adults aged 18 years or older: A randomised, double-blind, placebocontrolled, phase 2 trial. Lancet. 2020; 396: 479-488. https://doi. org/10.1016/S0140-6736(20)31208-3 